\title{
Los "Anales del Cuzco" y su presunto autor Don Diego de Esquivel y Navia
}

Contribución al esclarecimiento de los problemas que suscita una de las más valiosas fuentes de la Historia del Cusco

Nota previa.

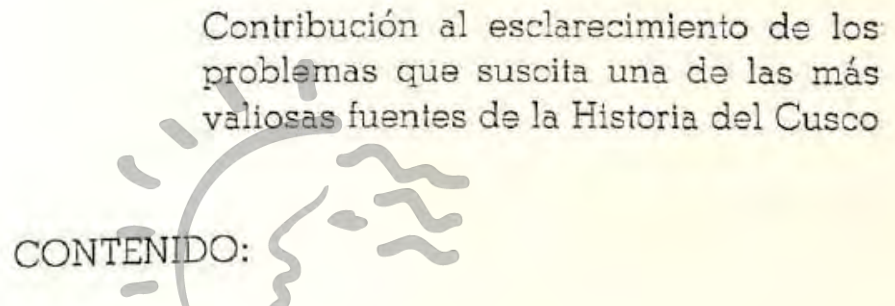

Datos biográficos de don Diego de Esquivel.

La Obra.

El manuscrito de la Obräblioteca de Letras

El autor.

¿Es Esquivel el verdadero autor de los "Anales?

Esquivel y otros historiadores.

Parágrafo final.

\section{NOTA PREVIA}

Durante el siglo XVIII se escribió en el Cusco una notable crónica histórica conocida entre nosotros con el nombre de ANALES DEL CUZCO, cuyo manuscrito, copiado más de una vez, ha sido atribuído a la pluma del ilustrado canónigo de la Catedral cuzqueña doctor don Diego de Esquivel y Navia, que viviera hasta 1779. Que éste sea el nombre del autor de los antiguos Anales, no ha podido comprobarse. Lo cierto es que la obra reune excepcional interés, de allí que nos hayamos propuesto estudiar los problemas referentes al autor y la curiosa historia de su viejo manuscrito. Tal el objeto del presente artículo. 
No tenemos, por supuesto, la presunción de haber agotado el tema. Al contrario, declaramos desde ahora que no ha sido posible dar término a nuestra averiguación, pues, lo investigado se atasca en un punto insuperable. No obstante, sin más vacilaciones, queremos ofrecerla ahora a la curiosidad de los lectores de esta importante Revista, seguros como estamos del interés de tan valioso asunto.

\section{DATOS BIOGRAFICOS DE DON DIEGO DE ESQUIVEL}

Son muy pocos los datos referentes a la vida de este preclaro cuzqueño que nos ha sido posible averiguar.

Sabemos que fué hijo del segundo marqués de Valleumbroso don Diego de Esquivel y Navia, de allí que llevara ese mismo nombre. Monseñor Juan $\AA$. Casanova, historiador bien informado, nos ha dicho que el doctor Esquivel era hijo natural del marqués y que el nombre de su madre corresponde al de doña Bernarda Mora.

Recordaremos que los cusqueños Esquivel eran descendientes directos del conquistador del Perú don Rodrigo de Esquivel y Cueva y que el bisnieto de éste, don Diego de Esquivel y Jarava, abuelo de nuestro analista, fué el primer marqués de San Lorenzo de Valleumbroso, desde 1687. Contrajo éste nupcias en 1669 con doña Guiomar de Navia Salas y Valdés, dama chuquisaqueña, y tuvo por hijo primogénito a don Diego de Esquivel y Navia, segundo marqués y padre de nuestro analista. Los Esquivel, como sabemos, cran poseedores de cuantiosa fortuna y en el Cuzco, es conocida la calle donde tuvieran su casa con el nombre de "Marqués", la misma que antes de que 21 Rey de España creara en 1687 el marquesado de Valleumbroso, se llamara "Calle Esquivel", por haber tenido allí siempre los Esquivel su casa residencial.

No hemos podido averiguar la fecha del nacimiento del presunto autor de los ANALES, apenas si sabemos que se dedicó desde joven a la carrera eclesiástica y que llegó pronto a descollar como una verdadera lumbrera. La verdad es que no debe haber sido corta la ilustración que adquiriera, pues, su obra demuestra la personalidad de un historiador erudito y consta, además en los ANALES que compró una valiosa biblioteca y que vivió enriqueciéndola constantemente.

Se ignora la época en que comenzara a escribir sus célebres Anales cuzqueños, pero, por su propia información sabemos que dió término a la primera parte en 1740 y a la segunda en 1750. El P. Rubén Vargas Ugarte nos dice que Esquivel concluyó la redacción de su obra en 1740 (1).

(1) Rubén Vargas Ugarte: "Historia del Perú.-Fuentes", Lima, 1939, p. 258. 
Esta fecha, como hemos dicho, sólo se refiere a la primera parte, pues, es erróneo afirmar que pudo ser concluída en 1740 una obra que narra sucesos acaecidos hasta 1749 .

Copias de documentos referentes al juicio de una capellanía existentes en la Biblioteca de la Universidad del Cuzco (Vol. № 091.1, primer protocolo) nos han llegado al conocimiento de que el doctor Esquivel en enero de 1777 era Provisor y Vicario General del Cabildo del Cuzco, cargo en el que, ese mismo año, fué reemplazado por el doctor don Juan Antonio Tristán.

Ms. Casanova ha completado estos datos diciéndonos que Esquivel fué Dean de 1778 a 1779 , año en que falleció.

\section{L.A OBRA}

ANALES DEL CUZCO debiera ser el nombre genérico de la obra de Esquivel, aunque con esta denominación sólo se conozca su segunda parte. Pues bien, estos Anales han sido divididos, como hemos visto, en dos partes. La primera, que D. Rícardo Palma bautizó con el nombre de "Noticias Cronológicas del Cuzco" y publicó en 1902, comienza desde la fundación de la monarquía incaica el año de 1043 y acaba el año de 1600, en plena etapa colonial.

Los capítulos de esta parte referentes a los Incas y, particularmente, aquellos párrafos que narran sus conquistas y describen el itinerario seguido en ellas, constituyen un resumen magnífico de lo que Garcilaso escribiera sobre las campañas de cada monarca en sus célebres "Comen. tarios Reales". Este autor era entonces la más insigne autoridad en el asunto, lo que explica elffenćmenón peto, (la Mistoría de cada Inca no se agota en la narración de sus conquistas sino es completada por Esquivel con una genealogía verdaderamente importante, cosecha personal de nuestro autor que constituye un valioso aporte histórico al mejor conocimiento de la descendencia de cada uno de los Incas (2).

Desde su proemio esta primer aparte denota la presencia de un autor bien informado y aunque predomina en ella un manifiesto respeto a las opiniones del Cronista Inca, hay que subrayar que en ningún momento se pierde la personalidad del autor que hasta corrige a los que consulta, como pasa con Gómara en la pág. 99, con Calancha en la pág. 212 y con el mismo Garcilaso en las pgs. 160, 165 y 212.

(2) Estas genealogías han sido utilizadas por H. H. Urteaga en su obra "El Imperio Incaico", Lima, 1931 Y, posiblemente, también por D. Justo Sahuaraura Inca, en su libro "Recuerdos de la Monarquía Peruana o Bosquejo de la Historia de los Incas". París, 1850. 
La redacción de esta primera parte, por declaración del mismo autor, fué acabada el día de la Pascua de Resurrección de 1740, y, como ya dijimos, se cierra con la narración de los hechos acaecidos en el Cusco durante el año de 1600.

La segunda parte, publicada en 1901 en tomo separado con el nombre de "Anales del Cuzco" 1600-1750, contiene todos los acontecimientos que el autor ha querido anotar acaecidos desde el año de 1601 hasta el de 1749. Naturalmente esta es la parte más interesante de la obra. No puede hallarse cuadro más completo de la vida colonial cusqueña que éste descrito con minuciosidad y lucido empeño por el doctor Esquivel. Son varias las obras que le sirven aquí de consulta; entre ellas destaca como más importante el famoso "Teatro Eclesiástico de Indias" que el maestro Gil Gonzáles Dávila escribiera en 1649 y 1656, pero, las mejores fuentes históricas de nuestro autor han sido indudablemente los libros de ambos Cabildos cusqueños y un gran número de documentos y papeles viejos que le han proporcionado fechas y datos muy valiosos. De no haber utilizado el Dean Esquivel estos documentos, hoy día ignoraríamos una gran parte de nuestra historia local.

Claro es que no faltan narraciones ociosas de hechos triviales que no interesan al historiador; cierto es, también, que son abundantísimos los relatos que se refieren a ceremonias y actos religiosos que eran rutina durante el período colonial, pero, si consideramos que el criterio de los hombres de ese tiempo no era el de nuestros días y que tanto por la condición del autor como por el espíritu de la época, las cuestiones religiosas tenían que ocupar en todo el plano más importante, habremos hallado la justa diśculpa de los defectoscanotados, si es que éstos puedan llamarse así.

Por otra parte la obra parece haber sido escrita para uso personal de su autor y no para la publicidad. Así parece desprenderse de los pasajes en que el autor narra hechos acaecidos en su época, hechos presenciados quizá por éli entonces, el libro se convierte casi en un diario de recuerdos personales. Pero si dejamos todo esto, y del cúmulo de noticias que trae el libro entresacamos con cuidado el cuadro propiamente histórico del Cuzco que el autor ha querido presentar, veremos la excepcional importancia que la obra reviste. Allí está el Cuzco colonial levantando iglesias y campanarios, fundando congregaciones y monasterios, fundiendo y consagrando campanas, enjoyando templos y celebrando suntuosas fiestas religiosas. Allí están también otros aspectos secundarios del verdadero Cusco colonial que no alcanzamos a ver en ninguna otra 
lectura. Por todo esto y mucho más la obra es incuestionablemente una de las más interesantes fuentes históricas de nuestra ciudad.

\section{EL MANUSCRITO DE LA OBRA}

Es posible que el manuscrito que se presume fuera dejado por Esquivel sufriera una verdadera dicotomía. Esta fué la razón por la cual Palma se vió precisado a publịcarlo por partes. Veremos cuáles son los motivos que nos asisten para sostener esta creencia.

En nuestra investigación alrededor del asunto hemos podido descubrir que uno de los que utilizaron la obra de Esquivel fué D. Pio Benigno Meza. Este escritor en sus "Anales" publicados en los años de 1866-67, demuestra muy a las claras haber conocido sólo la primera parte del manuscrito de Esquivel.

En cuanto a la parte segunda, hemos podido averiguar que la primera persona que llegó a utilizarla fué la señora Clorinda Matto de Turner en sus celebradas "Tradiciones Cuzqueñas". Del examen de lo publicado por esta escritora hemos sacado en limpio que pudo haber conocido sólo esta segunda parte, habiendo ignorado, posiblemente, la existencia de la primera.

Lo dicho hasta aquí parece ya indicarnos que, puesto que la obra fué utilizada por partes, el manuscrito original debió haber sufrido dicotomía desde muy temprano, mucho antes de que se hicieran copias de su texto, las cuales aparecen, razonablemente también separadas.

Ignoramos los motivos por los que esta segunda parte fué a parar a Lima; también ignoramos en qué tiempo fuéllevada desde el Cusco, pero, suponemos que en una de las varias 1 yeces quesfueron trasladados documentos y papeles antiguos el manuscrito fué a dar a la Biblioteca Nacional y, luego, a manos de su director D. Ricardo Palma, quien, seguro de su singular importancia lo hizo publicar en 1901 con el nombre de "Anales del Cuzco", 1600-1750. En los "Renglones Preliminares" que preceden a esta publicación Palma declara que "el cronicón por demás curioso y entretenido" pertenece a la Biblioteca de su cargo y da a entender claramente que había sido encontrado en ella y que desconocía el nombre de su autor.

Mientras tanto, desde antes de 1887, una copia del manuscrito co. rrespondiente a la primera parte actuaba en poder del canónigo de la Catedral del Cuzco Dr. Fernando Pacheco, quien la había adquirido de D. Emilio Montes, según lo cuenta el Dr. Mariano Gibaja (3). El canóni-

(3) Mariano C. Gibaja: "Influencia de la Iglesia en la Historia del Cuzco", 1919, p. 32. 
go Pacheco comenzó a publicar ese año esta parte de la obra, habiendo llegado a realizar la impresión de los siete primeros pliegos. Suspendida definitivamente la publicación, Pacheco obsequió la copia de su propiedad a la Biblioteca Nacional de Lima y, entonces, Palma pudo publicarla en 1902 con el nombre de "Noticias Cronológicas del Cuzco", exactamente un año después de publicada la segunda parie.

Parece que el señor Palma al editar esta última en 1901, no conocía todavía la copia de la parte primera que desde el Cusco enviara después el canónigo Pacheco a la Biblioteca Nacional. Así se colige de la lectura de sus "Renglones Preliminares" escritos en 1901.

Hasta aquí todo está perfectamente claro, pero luego viene algo que parece embrollar el asunto. El Dr. Mariano Gibaja en su tesis doctoral publicada en 1919, al referirse a las obras de Esquivel (Noticias Cronológicas y Anales del Cuzco) dice lo siguiente: "La primera comprende desde la fundación del imperio del Tahuantinsuyo en 1043 hasta fines de 1600, según el original manuscrito que posee el Dr. Fortunato L. Herrera... quien es sabedor de cómo un ejemplar mui mal copiado i truncados los años 1596, 97, 98, 99 y 1600, fué a dar a la Biblioteca Nacional de Lima y se imprimió con todos los defectos de la copia, insertado en el libro "Apuntes Históricos del Perú" que entre nosotros es mui conocido i leido". (Este ejemplar mal copiado y trunco de la primera parte es el manuscrito obsequiado por el canónigo Pacheco a la Biblioteca Nacional, según hemos visto ya).

"La segunda parte comprende desde 1600 hasta 1750. El manuscrito original, según consta al principio de la primeta obra impresa, la adquirió de poder del distinguido arqueólbogo Sr. Emilio, Montes el Sr. Canónigo Dr. Don Fernando Pacheco, quien después de publicar sólo los siete primeros pliegos, lo mandó a la Biblioteca Nacional de Lima, donde se publicó íntegra en 1902..." (4).

El primero de estos párrafos que pertenecen al doctor Gibaja está perfectamente claro y de acuerdo a la verdad, pero el último es un cúmulo de falsedades y contradicciones, peca de obscuro y puede ser entendido de las dos maneras siguientes: 10 Parece que se quisiera dar a entender que el manuscrito obsequiado por Pacheco a la Biblioteca Nacional es el original completo de la obra, no otra cosa dicen las palabras "original" e "íntegra". Pero, ya hemos visto, esto no puede ser más falso. El manuscrito obsequiado por Pacheco no es ni original ni completo, es la copia que precisamente Gibaja moteja de mal copiada y trun-

(4) Gibaja, Ob. Cit. p. Idem. 
ca en su primer párrafo y que por milagro de una lamentable equivocación Gibaja quiere, en su acápite siguiente, convertirla en original e íntegra. Para convencerse de ello no hay sino que leer el corto preliminar que Palma escribe al publicar el manuscrito de Pacheco con el nombre de "Noticias Cronológicas del Cuzco" en 1902.

$2^{\circ}$ Por otra parte, si Gibaja, en su segundo párrafo que comentamos, quisiera afirmar que el obsequio de Pacheco corresponde a la segunda parte de la obra, ya sabemos que eso signifcaría otra equivocación, pues, repetimos, Pacheco obsequió a la Biblioteca Nacional una copia de la primera, no de la segunda parte. Además, si Pacheco obsequió el manuscrito original a Lima, ¿cómo es posible que, por afirmación del mismo Gibaja en su primer párrafo, el doctor F. L. Herrera haya podido comprar en 1911 en el Cusco el original de la primera parte, tal como también afirma Gibaja?, ¿es que hubieron dos originales? El doctor Gibaja indudablemente se contradice; claro es que no era perito en el manejo de estas cosas o sólo escribió informándose verbalmente y mal, quizá sin haber visto las palabras preliminares que Palma escribiera en sus dos ediciones.

Así pues, aclarado este punto, seguimos creyendo que el manuscrito original sufrió dicotomia desde muy temprano. El P. Rubén Vargas Ugarte supone que la división de la obra en dos partes se debe a Palma (5), nosotros, por las razones ya expuestas, creemos que llegó a conocer una después de otra las partes del manuscrito, primero la segunda y después la primera, por eso es que se decidió involuntariamente a publicar la obra por partes, dando preferencia alla segunda. Por lo menos debe ser cierto el hebhogdelquecPalmbi sóloldespués de haber publicado en 1901 la segunda parte, pudo darse cuenta de que se trataba de una sola obra y sólo entonces se decidió a la publicación de la primera parte con el nombre de "Noticias Cronológicas", en 1902.

De otro modo, si recibió el manuscrito de la obra completa de manos de Pacheco, como parece insinuar Gibaja, cómo podremos explicarnos los motivos por los cuales nuestro tradicionista se vió precisado a publicar primero la segunda parte y después de un año la primera? Si poseyó al comienzo el manuscrito completo, ¿por qué no editó, como era razonable, toda la obra completa en una misma fecha o primero la primera parte y después la segunda?

Sigue en pie entonces nuestra creeancia de que el manuscrito original se separó desde muy temprano en dos partes.

(5) Rubén Vargas Ugarte, Ob. Cit. Pg. Idem. 


\section{EL ÄUTOR}

Así, como hemos visto, fué publicada la obra sin crue se pudiese en ese entonces saber el nombre de su autor. Apenas, por la lectura del texto, pudo Palma colegir que se trataba de un canónigo de la Catedral del Cuzco "que, de 1725 a 1750, empleó sus horas en compilar las noticias históricas compulsando las actas de ambos Cabildos y demás documentos de que pudo disponer" (6).

Es ya en 1911 cuando pudo averiguarse el presunto nombre, desconocido hasta entonces, de nuestro analista. Corresponde este descubrimiento a los doctores F. L. Herrera y Francisco Sivirichi, aunque el hallazgo realizado por ellos no se haya divulgado $\mathrm{y}$, a veces como en nuestro caso, cuesta verdadero trabajo averiguar la participación que estos investigadores tuvieron en el asunto.

El Dr. Gibaja nos dice a este respecto: "En 1911, al adquirir el original de la primera obra (Noticia Cronológica), los doctores F. L. Herrera y Francisco Sivirichi escribieron una carta al Sr. Palma, anunciándole que el autor de las Crónicas del Cuzco es el canónigo Dr. Diego Esquibel y Navia, cuya firma auténtica existía en dicho manuscrito. En respuesta el Sr. Palma dijo que él también se inclinaba por el mismo autor."

"Yo, de mi parte, he procurado examinar las actas capitulares de aquellas años, i me he informado que el Sr. Canónigo Esquivel i Navia fué todo un talento i de vasta ilustración, siendo su letra exactamente iqual en las actas como en el manuscrito i firma ya indicados" (7).

Estos datos aclaran perfectamente todo lozque sobre el descubrimiento del nombre do nuestrq autor queriamos averiguar, pero, sin duda por la poca difusión que alcanzó esta noticia, el nombre de Esquivel y Navia sigue ignorado.

Hemos de ver en seguida cómo Esquivel ha sido y sigue siendo objeto de dudas y de olvido.

En 1912 el Dr. Luis E. Valcárcel en su trabajo "Kon, Pachacamac, Uirakocha" afirma que el autor de las NOTICIAS CRONOLOGICAS es don Diego de Esquivel "según investigaciones últimas de los conocidos historiógrafos cuzqueños doctores Fortunato L. Herrera y Francisco'Sivirichi" (8). Por otra parte, en 1919, el mismo doctor Valcárcel aparece como dictaminador de la tesis tantas veces citada de Gibaja y, tanto por el dato anterior como por esta última circunstancia, sabemos que estaba per-

(6) Prólogo de los "Anales del Cuzco", 1901.

(7) Gibaja, Ob. Cit, pgs. 32-33.

(8) Revista Universitaria, Cuzco, Año I, No 3. Dic. 1912, pg. 3. 
fectamente informado que D. Diego de Esquivel es el aufor de la obra que nos ocupa. Sin embargo, en 1935 Valcárcel se ha olvidado completamente del nombre de nuostro analista y vuelve a mencionar sus NOTICIASS CRONOLOGICAS atribuyéndolas a autor anónimo (9).

Suponemos que el doctor Valcárcel tenga motivos que justifiquen este olvido que nosotros interpretamos como un cambio de opinión. Posiblemente abriga dudas respecto de la paternidad de la obra. Y en este punto no debe andar muy alejado de las opiniones muy personales del historiógrafo cusqueño Ms. Juan A. Casanova, opiniones llenas del mayor interés por lo que nos permitimos incluirlas en esta parte de nuestro estudio.

Ms. Casanova nos dice que durante cierto tiempo llegó a poseer el manuscrito que después compraron los Drs. Sivirichi y Herrera. Que este manuscrito, considerado por éstos como el códice original, lleva el nombre de D. Diego de Esquivel y que su contenido parecía escrito por este mismo, aunque esto último no le parece muy seguro. Que bien puede ser Esquivel el autor de la obra, pero que hay varios motivos que le hacen abrigar dudas. Esas dudas se basan principalmente en la lectura del manuscrito que, lamentablemente, nosotros no hemos podido conocer y en otros detalles cuya discusión interesará mucho a nuestros lectores cuando haya llegado la odasión oportuna.

Aunque no decididamente, se inclina Ms. Casanova a creer que el Códice de los Anales puede ser obra de varios autores, quizá de varios religiosos, elaborada poco a. poco. Cree además posible que Esquivel no sea sino un copista delos varios que pudieron realizar copias del antiguo códice original, (puesj el manuscrito den propiedaà del Dr. Herrera no indica de manera precisa el nombre de su autor. Cree también que puede ser posible que Esquivel dejara su nombre en el manuscrito porque era su dueño y no porque era su autor, como era costumbre suya hacerlo con todas las obras antiguas que formaron parte de su biblioteca.

Como vemos, Ms. Casanova sólo da por aceptado lo que pueda demostrarse inconcusamente y la verdad es que en el actual estado de nuestra investigación esta clase de demostración no es posible. Sin embargo, existen elementos de prueba que son decididamente favorables a Esquivel y a ellos vamos a recurrir en el curso de nuestro trabajo para ver por qué no participamos de las dudas de Ms. Casanova.

Es posible que durante algún tiempo también el doctor F. L. Herrera tuviera dudas semejantes. Descubridor de Esquivel en 1911, parece

(9) Revista del Museo Necional. Lima, Tcmo IV, No 2, II Sem. 1935, pg. 176. 
haberlo olvidado en 1913, y los ANALES DEL CUZCO en el trabajo "Universidad de San Antonio Abad" (10) que escribiera dos años después de su descubrimiento, son mencionados como de autor anónimo, y no se diga que Herrera no tenía que saber que el autor de las NOTICIAS CRONOLOGICAS y de los ANALES es una misma persona, pues, Palma lo afirma ya desde 1902 (11). Este curioso caso parece indicar una distracción del Dr. Herrera, pues, en 1914 se ratifica en el conocimiento de Esquivel como autor de los ANALES (12), iqual que lo había hecho siempre su codescubridor Dr. Sivirichi y, con ellos, los Drs. Horacio H. Urteaga (13) y Carlos A. Romero. Precisamente fué este último quien se encargó de completar la publicación de la obra incluyendo en las páginas de la REVISTA HISTORICA la copia de la parte inédita de los Anales que, extractada del manuscrito original, enviaran a Lima los Drs. Herrera y Sivirichi (14).

\section{¿ES ESQUIVEL EL VERDADERO AUUTOR DE LOS "ANALES"?}

Ya hemos dicho que no es posible dar definitiva respuesta a esta pregunta. Apenas si nuestro estudio alcanza a proporcionarnos algunos datos que no tienen categoría de evidencias y que nos han decidido a participar, con algunos investigadores informados sobre este particular, de la creencia de que sea Esquivel el verdadero autor de los antiguos ANALES. Para colmo de infortunio no nos ha sido posible estudiar el manuscrito que perteneciera al Dr. Herrera, considerado como original. Sabemos que quien 10 pospe ahroranes el DreLuis E. Valcárcel, a quien, lamentablemente, no alcanzamos a ver en nuestra ultima visita a Lima. Ia lectura del citado manteerito nos habria sido de gran valor en el caso presente. No obstante esta circunstancia, nuestra dificultosa averiguación arroja numerosos datos favorables a Esquivel, de los cuales sólo vamos a mencionat los siguientes:

1. El plan de la obra tan bien llevado a término y el estilo parecen corresponder claramente a un solo autor y no a varias;

$2^{\circ}$ Las citas bibliográficas demuestran una cultura pareja en toda la extensión de la obra, lo que denuncia a un solo autor;

(10) Rev. Universitaria, Cuzco, Año II, No 6, Set. 1913, pg. 36.

(11) Palabras liminares escritas por Palma en las "Noticias Cronológicas", 1902.

(12) Rev. Universitaria, Guzco, Año III, N9 11, Dic. 1914, pg. 4.

(13) Horacio H. Urteaga, Ob, Cit. pg. 115

(14) Revista Histórica, Tomo V, 1913, pgs. 209-224. 
$3^{\circ}$ La obra, a las claras, ha sido escrita por un historiador de vocación que estudió y utilizó valiosas fuentes; tan bien informado que, como hemos visto, hasta se permitió corregir a Garcilaso. Varias personas no habrían podido tratar el asunto y llevarlo a término con igual dedicación y amor y, sobre todo, con igual ilustración;

$4^{\circ}$ La comparasión caligráfica realizada por el doctor Gibaja, descrita en su tantas veces citada tesis;

$5^{\circ}$ El hecho de que la obra abunda en detalles referentes a los Esquivel y en particular al Dean Esquivel, lo que un extraño no hubiera podido anotar por no conocerlos. Entre estos detalles tenemos la compra de una biblioteca; las palabras textuales que pronunció el mismo Esquivel en uno de los incidentes de la compra" ( $2^{\alpha}$ parte, ps. 339 y 340 ) y, sobre todo, la minuciosidad en anotar su participación en numerosas ceremonias religiosas como solo puede hacerlo uno mismo.

Todas estas circunstancias nos sosfienen en la creencia de que D. Diego de Esquivel es el verdadero autor de las Crónicas que estudiamos. Esta creencia se afirma mucho más si recordamos que el célebre Dean cuzqueño fué uno de los hombros más lustrados en el Perú de su tiempo.

Menos razones que las anteriormente enumeradas han servido a varios investigadores para atribuir al P. Blas Valera la paternidad de la "Relación Anónima" que don Marcos Jiménez de la Espada publicada en 1879.

\section{ESQUIVEL Y OTROS HISTORIADORES}

No hemos querido dar têminf al estudio presente sin dedicar, aunque fuera pocas líneas, od ga influericia dela jobrar esquiveliana en la de otros historiadores. Y así lo haremos, aunque por esta vez sólo nos referiremos a los casos de D. Pio Benigno Meza y de doña Clorinda Matto de Tumer, mencionados al comienzo de nuestro artículo.

El del señor Meza es un caso verdaderamente curioso cuyo descubrimiento y estudio nos pertenece. Nadie en más de cuarenta años había tenido la curiosidad de comparar las Crónicas de Esquivel concluídas en 1750 con los "Anales de la Ciudad del Cuzco" del señor Meza, publicados allá por los años de 1866 y 1867 . El Dr. Herrera, que conocía la obra de ambos, era seguramente, el más indicado para esa labor (15); tampoco alcanzó a realizarla el pəruanista Tschudi, quien se limita a expresar su gesto despectivo hacia los Anales del señor Meza. Lo cierto es que este último poseyó un manuscrito idéntico en texto al que nosotros hemos estudiado como obra del Dr. Esquivel, que suponemos puede ser

(15) Rev. Universitaria, Cuzco. Año III, No 9, junio 1914, pq. 53, llamada (3). 
una copia bastante mejorada de la oue sirvió a Palma para publicar las NOTICIAS CRONOLOGICAS.

El señor Meza utilizó este manuscrito en forma bastante exagerada, tanto que el primer tomo de sus celebrados "Anales de la Ciudad..." constituyen casi una primera edición de ese manuscrito que, como hemos visto, según opiniones autorizadas, pertenece en su origina a la pluma del Dr. Esquivel.

Hemos encontrado en los tomos $1^{\circ}$ y $2^{\circ}$ de la obra de Maza, 47 y 19 párrafos, respectivamente, es decir 65 en total, tomados en su mayor parte casi ad litteram de la obra de Esquivel, 65 párrafos de extensión varia que pertenecen a Esquivel y que han sido incluídos por Meza en su obra como suyos (1).

Es inútil subrayar que este autor para nada menciona la existencia del tal manuscrito. Sería, por supuesto, ocioso hacer una lista de la numeración de las páginas que llevan tal copia o hacer la reproducción de tales párrafos, pues, necesitaríamos para ello un espacio enorme. Pero, no es sólo esto; hay mucho mas. El título de la obra y el dé todos y cada uno de los capítulos, peftenecen a Esquivel; el orden observado en la mención de personajes o de cualesquiera nombres juntos, como en las listas de la descendencia de cacla Inca y en las de lugares, pueblos o tribus, también pertenece a Esquivel; casi todas las citas de autores coinciden en oportunidad y son las mismas en ambos escritores, al iqual que la reproducción de los mismos pasajes tomados, por lo general, de los mismos autores. Algo más, en la obra de Meza se nota un estilo desigual, con relativa frecuenclaspues, cparedel quelen a toda costa de injertar el antiguo estilo de Esquivel en el suyo. Esto puede notarse claramente en muchas partes donde Meza parece cansarse de coplar servilmente el manuscrito y trata de hacer suyo lo de Esquivel cambiando la forma de dicción y el orden expresivo de los vocablos para disimular el estilo un tanto anticuado del otro. Nada diremos o casi nada del orden expositivo de la obra, es decir, de la disposición de sus capítulos y de sus párrafos; del plan general de la obra y de sus ideas de detalle, porque, especialmente en el primer tomo, casi íntegramente todo pertenece a Esquivel; hasta las citas de simple adorno.

Pero, lo más interesante es que el señor Meza, después de un año de publicado su primer tomo, al dar a publicidad el segundo; allí donde parece querer abandonar completamente al antiguo manuscrito, donde hace gala de lo suyo, reproduce en la página 261, como perdido en un océano, un corto pasaje de la obra de Esquivel que dice pertenece a "un antiguo R. P. cuzqueño" a cuyos "apuntes manuscritos muy curiosos" pa- 
rece no querer prestar mayor atención. Y así al fin, como vencido por un íntimo remordimiento declara Meza la existencia del manuscrito que, sin decirlo, utiliza en casi toda la extensión de sus Anales. ¿Cómo sabía Meza que el autor del manuscrito era un religioso?, ¿conocía quizá su nombre? No hemos podido averiguarlo, lo que sí sabemos es que nuestros lectores sabrán juzgar con justicia este caso.

En cuanto a la señora Matto de Turner, la situación es diferente. Esta distinguida escritora cuzqueña goza de prestigio preferentemente por haber publicado una colección de 54 Tradiciones en dos series que, como sabemos, constituyen una parte muy interesante de nuestro acervo tradicional. Pues bien, para escribirlas, la señora Matto tuvo que consultar diferentes fuentes históricas, pero la principal, la que mayor material le proporcionó para la redacción de sus sabrosos relatos es la obra de D. Diego de Esquivel. La reproducción entre comillas que hace nuestra tradicionista, de varios pasajes pertenecientes a este escritor en más de seis tradiciones, nos ha convencido de que conoció la obra de aquél todavía inédita en ese tiempo. Además, la señora Matto menciona con frecuencia un antiguo manuscrito que le sirve para sus relatos y lo llama "Chrónica Historial" en más de una oportunidad. Esta Crónica fué una copia del manuscrito de Esquivel, pues los pasajes reproducidos están en el texto de los Anales, excepción hecha de uno solo que figura en la tradición "Depósito Parlante", que narra un episodio del año 1543 y que no hemos podido encontrar en la obra de Esquivel.

Pero hay otro asuntol qute nos hanllamada ja atención. La señora Matto en su tradición "El Bfazo Negro del Corregidor" reproduce un pa saje que claramente pettenece a Esquivel y que afirma haberlo tomado de la "Crónica Particular" manuscrita de un Padre Otomendi. No hemos podido averiguar si efectivamente este religioso escribió tal crónica, solamente estamos seguros que el tal episodio está narrado también por Esquivel en las páginas 175 y 176 de sus Anales y, por ello nos parece posible que la señora Matto creyó que la obra de Esquivel pertenecía al padre Otomendi. ¿No creerán nuestros lectores que esto último puede significar una nueva interrogación en el problema de la tan discutida paternidad de los ANALES?

Para finalizar y como comprobación de haber encontrado la principal fuente documental de la señora Matto diremos que de sus 54 tradiciones, 33 están basadas en pasajes históricos narrados en la obra de Esquivel. Vamos a indicar a continuación, el nombre de estas tradiciones y, entre paréntesis, las páginas de los Anales donde pueden ser encontrados los datos históricos en que se basan. 
Primera Serie: Lo que costó un recibimiento (p.2); El que manda, manda (p.46); Vaya un decreto (p.2); Treinta y nueve onzas de oro por cinco zedas y una sandía (ps.415-416); Cuenta clara (p.201); La Cruz de SacsayHuamán (ps.206-207); No hay Pedro Bueno (p.3); Veinticinco por la golilla (ps.37-38); El marqués de la Viga (p.67); El brazo negro del Corregidor (ps.175-176); La mala Carranza (p.173); Santa Catalina del Cuzco (ps.102115-116); Un hallazgo oportuno p.180); El Zelenque (ps.34-35-36); Los siete cajones (p.162); Arco Punco y su cruz (ps.119-120); Lo de antaño (p.186); Fundición de la María Angola (ps.128-129-131); Así paga el diablo a quien bien le sirve (p.222); La Virgen de los Remedios (ps.89-90); Lo que costaba una cauda (p.41); Tambo de Montero (p.83); Fué un milagro (ps.15-16); Año dos (ps. 155-257); segunda serie: Las tres hermanas (ps. 152-153); Caer a hora (p.40); Pobre importuno, saca mendrugo (p.145); Azotaina mayúscuya (p/200); Buena laya de hombre (p.142); El Risco de Yaya-Huarcusca (p.101); Plagas humanas (ps.244-245); Llamada del sepulcro (ps.250-251); De llama y fuego (p. 166).

\section{PARAGRAFO FINAL}

Que nuestro trabajo deja varias interrogantes sin respuesta no lo dudamos, mas nosotros creemos haber exhibido un fruto de perseverancia cosechado en el terreno más estéril de nuestra bibliografía local y en las más adversas condiciones que se puedan imaginar. No obstante lo mezquino de ese fruto, no estamos desanimados. Seguiremos trabajando en la búsqueda de las definitivas solueiones! diala sea con más fortuna que ahora. "Jorge Puccinelli Converso» 